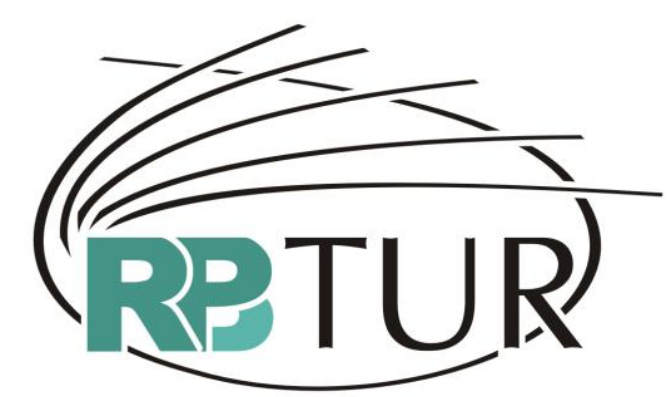

REVISTA BRASILEIRA DE PESQUISA EM TURISMO

\title{
LAS NUEVAS FORMAS DE TURISMO: CAUSAS Y CARACTERÍSTICAS
}

\author{
AS NOVAS FORMAS DE TURISMO: CAUSAS E \\ CARACTERÍSTICAS
}

\section{NEW TOURISM: CAUSES AND CHARACTERISTICS}

\author{
Alberto JonayRodriguez Darias ${ }^{1}$ \\ Agustín Santana Talavera² \\ Pablo Diaz Rodriguez ${ }^{3}$
}

\begin{abstract}
Resumen: El turismo a nivel internacional ha pasado por importantes cambios, entre ellos el que se produjo a mediados del siglo XX y dio lugar al sistema turístico tal y como lo conocemos. Sin embargo, en las últimas dos décadas parece que el escenario se modifica a mayor velocidad; la aparición de lo que a mitad de la década de los 90 se denominaron "nuevas formas de turismo" ha cambiado las reglas del juego en múltiples sentidos y perspectivas, abriendo al Planeta como destino y consolidando el "todo puede ser vendido a un turista". Este trabajo trata de determinar las causas de estas modificaciones e incitar el debate basado en las relaciones de aquellas "nuevas formas de turismo" y las que surgen en la actualidad.
\end{abstract}

Palabras clave: Estudios Sociales del Turismo. Nuevas Formas de Turismo. Sostenibilidad. Autenticidad. Experiencia Turística.

Resumo: O turismo internacional passou por grandes mudanças, dentre as quais a acontecida em meados do século XX que resultaram nas formas conhecidas de turismo. No entanto, nas últimas duas décadas as mudanças parecem ter se

${ }^{1}$ Doutorando no Laboratório de Antropologia Social da Universidad de La Laguna, Tenerife, Espanha. Email: jonayalberto@yahoo.es

2 Doutor em Antropologia Social, professor titular da Universidad de La Laguna (Tenerife, Espanha) e subdiretor do Instituto de Ciências Políticas e Sociais da mesma instituição.Tem atuado nas linhas de pesquisa da Antropologia do Turismo e do Patrimônio Cultural. É fundador e editor de PASOS - Revista de Turismo y Patrimonio Cultural (www.pasosonline.org), revista eletrônica internacional e de caráter multidisciplinar. Email: asantana@ull.es

3 Mestre em Antropologia e doutorando pesquisador da Universidade Complutense de Madri, onde estuda questões relacionadas a meio ambiente, turismo e ordenamento territorial. Email: diazrodriguez@gmail.com 
acelerado. O surgimento das chamadas "novas formas de turismo" nos anos 1990 mudou as regras, tanto em sentido quanto em perspectiva, abrindo o planeta enquanto destino turístico e consolidando que "qualquer coisa pode ser vendida para um turista". Esta pesquisa busca determinar o que ocasionou estas mudanças e iniciar um debate relacionando aquelas "novas formas de turismo" com as atuais.

Palavras-chave: Estudos Sociais do Turismo. Novas Formas de Turismo. Sustentabilidade. Autenticidade. Experiência Turística.

Abstract: International tourism has been through major changes, one of them by the 1950 s which resulted in conventional forms of tourism. Nevertheless, in the past two decades changes seem to go faster. The upsurge of what was labeled as "new forms of tourism" in the 1990s changed the rules in several ways, turning the planet into a tourist destination and consolidating that "anything can be sold for a tourist". This paper intends to determine which caused these changes and begin a debate related to those (old) new forms of tourism and contemporary new forms of tourism.

Keywords: Social Studies in Tourism. New Forms of Tourism. Authenticity. Touristic Experience.

\section{Introducción}

Las llamadas "nuevas formas de turismo" aparecen con fuerza en los primeros años de la década de 1990 a la saga de productos que combinaban bienes, recursos y experiencias en un territorio dado, desarrollándose hasta nuestros días (en los que no podemos decir que sean tan nuevas). Turismo solidario, responsable, sostenible, verde, comunitario, de aventura, ecoturismo, agroturismo, etc. a nivel general, pueden caracterizarse por constituir productos alternativos o complementarios, según el caso y punto de vista, a los tradicionales de sol y playa, balnearios o termas, y suelen ofrecerse como aparentemente regidos por criterios de sostenibilidad, satisfactores de nuevas demandas, relacionados con reivindicaciones de prestigio social $y$, en ciertas ocasiones, con ritos de paso, incluyendo en el elenco de elementos explotables turísticamente algunos aspectos no utilizados hasta ese momento (desde inocuos como granjas - agroturismo - a horripilantes como espacios devastados por la guerra - reality tourism).

En estas páginas se determinarán las principales causas que dieron lugar a estas modificaciones en el sistema turístico y que constituyeron, desde nuestra consideración, una renovación del mismo y sus procesos asociados. 


\section{Acerca de la Aparición de las Denominadas Nuevas Formas de Turismo}

La explicación más aceptada para la aparición de lo que se denominó "nuevas formas de turismo" se basa en (a) instauración del paradigma de la sostenibilidad, (b) su acomodación en los discursos respecto al turismo y (c) la concienciación de los ciudadanos, la difusión de las políticas, el marketing y, a veces, las prácticas turísticas en concordancia con él (Butler, 1994; Eadington y Smith, 1994; Ceballos-Lascuráin, 1996; Marín y Mendaro, 1996; Mora, Duch Devesa y Córdova Leiva, 2001; Smith, 2001c; Epler Wood, 2002; Drumm y Moore, 2002; Manwa, 2003; Santana, 2003, 2008; Valdés, 2004; Hernández Cruz, Bello Baltasar, Montoya Gómez y Estrada Lugo, 2005; Cruz Vareiro y Cadima Ribeiro, 2007; Duterme, 2007; Puertas Cañaveral y Paniza Prados, 2007; Stronza y Gordillo, 2008). Así se observa que:

(a) Si bien los inicios del movimiento conservacionista están íntimamente ligados con discursos provenientes del pensamiento ilustrado dieciochesco que reivindican el paisaje y la montaña de forma romántica frente al capitalismo incipiente, en el presente marco teórico se tomará como referencia el renacimiento que sufre éste tras la Segunda Guerra Mundial. Momento en que se establecen, en los países occidentales, modelos de producción que materializan la superioridad de la humanidad sobre la naturaleza con una intensidad sin precedentes.

Especialmente en Estados Unidos, se produjo un extraordinario crecimiento económico que facilitó una esfera de optimismo respecto a aquéllos. Incluso, a través de la concepción denominada desarrollismo (Gimeno y Monreal, 1999; Viola Recasens, 2004), se planteó que la exportación de estos modelos no sólo era posible, sino además deseable; se concibió, de manera absolutamente idealizada, como el modo de conseguir altos niveles de bienestar para la población mundial.

De acuerdo con la Comisión Mundial sobre el Medio Ambiente y el Desarrollo (1989, pp.21) una de las principales circunstancias que conducen a la modificación de este paradigma está relacionada con los viajes espaciales y 
la difusión de imágenes del planeta Tierra desde el exterior; con la consecuente constatación de su fragilidad y no centralidad en el universo.

En cualquier caso, a partir de los años setenta, frente a los planteamientos productivistas y del desarrollo que se habían extendido en décadas anteriores, aparecen nuevas concepciones (Valdés, 2004) que reconocen de manera más o menos decidida (1) las implicaciones negativas sobre el medio de este modelo y (2) la imposibilidad de exportación del modelo (pues el desarrollo de unos precisa del subdesarrollo de otros).

El devenir de este movimiento se materializa a través de eventos de carácter internacional (tabla 1), fundamentalmente propuestos por las Naciones Unidas.

\begin{tabular}{|c|c|c|c|}
\hline Evento o documento & Año & $\begin{array}{l}\text { Organismo } \\
\text { promotor }\end{array}$ & Ciudad \\
\hline $\begin{array}{l}\text { Aparece la Unión Mundial para la } \\
\text { Naturaleza (UICN) }\end{array}$ & 1948 & ONU & $\begin{array}{l}\text { Fontainebleu } \\
\text { (Francia) }\end{array}$ \\
\hline $\begin{array}{l}\text { Publicación del primer informe del } \\
\text { Club de Roma (Informe Meadows) }\end{array}$ & 1972 & Club de Roma & $\begin{array}{l}\text { Roma } \\
\text { (Italia) }\end{array}$ \\
\hline $\begin{array}{l}\text { I Conferencia de las Naciones Unidas } \\
\text { sobre el Medio Ambiente Global }\end{array}$ & 1972 & $\begin{array}{l}\text { UNESCO, } \\
\text { ONU, UICN y } \\
\text { WWF }\end{array}$ & $\begin{array}{l}\text { Estocolmo } \\
\text { (Suecia) }\end{array}$ \\
\hline $\begin{array}{lccr}\text { Aparición del Programa de } & \text { las } \\
\text { Naciones Unidas para } & \text { el } \\
\text { Medioambiente (UNEP) } & & \end{array}$ & 1972 & ONU & $\begin{array}{l}\text { Estocolmo } \\
\text { (Suecia) }\end{array}$ \\
\hline $\begin{array}{l}\text { Convenio sobre la Protección del } \\
\text { Patrimonio Mundial, Cultural y Natural }\end{array}$ & 1972 & UNESCO & $\begin{array}{l}\text { París } \\
\text { (Francia) }\end{array}$ \\
\hline $\begin{array}{l}\text { Conferencia de Naciones Unidas sobre } \\
\text { población mundial }\end{array}$ & 1974 & ONU & $\begin{array}{l}\text { Cocoyoc } \\
\text { (Méjico) }\end{array}$ \\
\hline $\begin{array}{l}\text { La Convención sobre } \text { los Humedales } \\
\text { de Importancia } \\
\text { (Convenio de Ramsar) }\end{array}$ & 1975 & UIVN & $\begin{array}{l}\text { Rámsar } \\
\text { (Irán) }\end{array}$ \\
\hline $\begin{array}{l}\text { Plan de Acción para la protección y el } \\
\text { desarrollo de la cuenca del } \\
\text { Mediterráneo }\end{array}$ & 1975 & ONU, UE & \\
\hline $\begin{array}{l}\text { Estrategia } \\
\text { Conservación }\end{array}$ & 1980 & $\begin{array}{l}\text { IUCN, WWF Y } \\
\text { UNEP }\end{array}$ & $\begin{array}{l}\text { Gland } \\
\text { (Suiza) }\end{array}$ \\
\hline Carta Mundial para la Naturaleza & 1982 & UICN & \\
\hline
\end{tabular}




\begin{tabular}{|c|c|c|c|}
\hline $\begin{array}{l}\text { Publicación del Informe de la } \\
\text { Comisión Mundial sobre el Medio } \\
\text { Ambiente y el Desarrollo "Nuestro } \\
\text { Futuro Común" (Informe Brundtland) }\end{array}$ & 1987 & ONU & \\
\hline $\begin{array}{l}\text { Conferencia de Naciones Unidas sobre } \\
\text { el Medio Ambiente y el Desarrollo } \\
\text { (Cumbre de Rio) }\end{array}$ & 1992 & ONU & $\begin{array}{l}\text { Rio de Janeiro } \\
\text { (Brasil) }\end{array}$ \\
\hline $\begin{array}{l}\text { Publicación del documento "Cuidar la } \\
\text { Tierra" }\end{array}$ & 1993 & $\begin{array}{l}\text { UICN, WWF y } \\
\text { ONU }\end{array}$ & \\
\hline II Cumbre de la Tierra & 1997 & ONU & $\begin{array}{l}\text { Nueva York } \\
\text { (EEUU) }\end{array}$ \\
\hline Cumbre del Clima y protocolo de Kioto & 1997 & ONU & $\begin{array}{l}\text { Kioto } \\
\text { (Japón) }\end{array}$ \\
\hline $\begin{array}{l}\text { Conferencia de Naciones Unidas sobre } \\
\text { cambio climático }\end{array}$ & 2000 & ONU & $\begin{array}{l}\text { La Haya } \\
\text { (Holanda) }\end{array}$ \\
\hline $\begin{array}{l}\text { Cumbre Mundial sobre el Desarrollo } \\
\text { Sostenible }\end{array}$ & 2002 & ONU & $\begin{array}{l}\text { Johannesburg } \\
\text { o } \\
\text { (Sudáfrica) }\end{array}$ \\
\hline Objetivos de desarrollo del Milenio & 2002 & ONU & \\
\hline $\begin{array}{l}\text { Redefinición de los objetivos de } \\
\text { desarrollo del Milenio }\end{array}$ & 2008 & ONU & $\begin{array}{l}\text { Nueva York } \\
\text { (EEUU) }\end{array}$ \\
\hline
\end{tabular}

Tabla 1 - Listado no exhaustivo de eventos relacionados con el cambio de paradigma respecto al desarrollo

Fuente: elaboración propia

Puede destacarse la fundación de la Unión Internacional para la Conservación de la Naturaleza (UICN) en el seno de las Naciones Unidas en 1948, como el principal organismo a nivel mundial en la búsqueda de formas sustentables de desarrollo. Su objetivo fundamental es "influenciar, motivar y asistir a las sociedades a través del mundo para conservar la integridad y diversidad de la naturaleza y garantizar que cualquier uso de los recursos naturales será equitativo y ecológicamente sostenible" (Vidaurreta Campillo, 2003).

1972 se constituye como una año clave para este movimiento; tiene lugar en Estocolmo la I Conferencia de Naciones Unidas sobre el Medioambiente Global (cuyo principal mérito consiste en mostrar que las cuestiones ambientales podían gozar de cierto val político), aparece el Informe Meadows en el ámbito en el ámbito del club de Roma (que llama la atención 
sobre la necesidad de impone límites al crecimiento), se crea el Programa de las Naciones Unidas para el Medioambiente (UNEP) y se firma en París el convenio sobre Patrimonio Mundial, Cultural y Natural (que supone el primer compromiso para la conservación a nivel internacional de la naturaleza y la cultura) promovido por la UNESCO. Y sólo dos años después, en el ámbito de la Conferencia de Naciones Unidas sobre Población Mundial, se promueve decididamente el concepto "ecodesarrollo".

Por su parte, la Estrategia Mundial para la Conservación planteó que el desarrollo se debe concebir como "la modificación de la Biosfera y la aplicación de los recursos humanos y financieros, tanto bióticos y abióticos, para la satisfacción de las necesidades humanas y el mejoramiento de la calidad de vida" (citado en Salinas Chávez y Osorio, 2006, pp.203).

Asimismo, es de gran relevancia la publicación, en 1987, del Informe de la Comisión Mundial sobre el Medio Ambiente y el Desarrollo "Nuestro Futuro Común", más conocido como informe Brundtland, que aporta el concepto "desarrollo sostenible"; aquel que "satisfaga las necesidades del presente sin comprometer la capacidad de las futuras generaciones para satisfacer las propias" (Comisión Mundial sobre el Medio Ambiente y el Desarrollo, 1989, pp.29).

La Conferencia de Naciones Unidas sobre el Medio Ambiente y el Desarrollo (más conocida como la Cumbre de la Tierra), celebrada en Río, constituye uno de los eventos más influyentes de este paradigma; en ella se demanda un reparto más equitativo de los recursos, la inclusión de las voces locales en los procesos de conservación de la naturaleza, la necesidad de transmitir a la sociedad los principios conservacionistas, se apuesta por una nueva estrategia ante el nuevo siglo (Agendas XXI) y se le otorga una gran difusión al concepto de sostenibilidad, asentándolo en las administraciones públicas.

1997 puede ser considerado también un año relevante en relación con este movimiento, ya que se celebró (1) en Nueva York una nueva Conferencia de las Naciones Unidas (II Cumbre de la Tierra) con el objetivo de insistir en lo 
planteado en Río hacía cinco años (constatando lo poco que se había avanzado desde entonces) y (2) la Cumbre del Clima, de la que surge el protocolo de Kioto (cuyo impacto sería evaluado en la Conferencia de La Haya sobre Cambio Climático). Así como 2002, año en el que se desarrolla la Cumbre Mundial sobre Desarrollo Sostenible de Johannesburgo y se establecen los Objetivos del Milenio (redefinidos en 2008).

(b) Las formas de producción y consumo relacionadas con el turismo no sólo no han quedado al margen de este paradigma, sino que es en su ámbito en el que más actividad se ha desarrollado, tanto a nivel teórico como en la aplicación (Instituto de Estudios Turísticos, 1997; Mora, Duch Devesa y Córdova Leiva, 2001).

Los acercamientos teóricos al turismo, al menos en el ámbito de las ciencias sociales, están marcados por la evolución de los paradigmas del desarrollo (Santana Turégano, 2007); de forma que en las décadas de los cincuenta y sesenta se destacan los factores positivos del desarrollo turístico (plataforma apologética), la de los setenta se caracteriza por la crítica sobre éste (plataforma precautoria), la de los ochenta por la búsqueda de mecanismos que hicieran del turismo una actividad sostenible (plataforma adaptativa), en los noventa se intenta establecer un cuerpo sistemático de conocimiento en torno al turismo (plataforma científico-céntrica) y en la primera década del siglo veintiuno encontramos la reiteración de la necesidad de aumentar ese cuerpo de conocimientos a favor (a) del interés público (plataforma del interés público) y (b) de la capacidad de predicción y adaptación a los posibles cambios (Jafari, 1994, 2005; Santana, 2009).

Por otro lado, si bien entre los eventos y documentos de carácter general citados anteriormente se hace referencia al turismo (especialmente a través de las Agendas XXI y la Cumbre Mundial sobre el Desarrollo Sostenible de Johannesburgo) puede encontrarse una multitud dedicados específicamente a la sostenibilidad en el ámbito de esta actividad (tabla 2). 


\begin{tabular}{|c|c|c|c|}
\hline Evento o documento & Año & $\begin{array}{l}\text { Organismos } \\
\text { promotores }\end{array}$ & Lugar \\
\hline $\begin{array}{l}\text { Declaración de Manila sobre el } \\
\text { turismo mundial }\end{array}$ & 1980 & OMT & $\begin{array}{l}\text { Manila } \\
\text { (Filipinas) }\end{array}$ \\
\hline Declaración de Acapulco & 1982 & OMT & $\begin{array}{l}\text { Acapulco } \\
\text { (Méjico) }\end{array}$ \\
\hline $\begin{array}{l}\text { Carta del turismo y código del } \\
\text { turista }\end{array}$ & 1985 & OMT & $\begin{array}{l}\text { Sofía } \\
\text { (Grecia) }\end{array}$ \\
\hline $\begin{array}{l}\text { Conferencia Interparlamentaria } \\
\text { sobre Turismo }\end{array}$ & 1989 & OMT & $\begin{array}{l}\text { La Haya } \\
\text { (Holanda) }\end{array}$ \\
\hline Loving them to death? & 1991 & Europarc & \\
\hline Turismo cualitativo & 1991 & $\begin{array}{l}\text { Asociación } \\
\text { Internacional } \\
\text { de Expertos } \\
\text { Científicos del } \\
\text { Turismo } \\
\text { (AIEST) }\end{array}$ & \\
\hline $\begin{array}{l}\text { Beyond the Green Horizon: } \\
\text { Principles of Sustainable Tourism }\end{array}$ & 1992 & WWF & $\begin{array}{l}\text { Londres } \\
\text { (Inglaterra) }\end{array}$ \\
\hline Carta del turismo sostenible & 1995 & OMT & $\begin{array}{l}\text { Lanzarote } \\
\text { (España) }\end{array}$ \\
\hline $\begin{array}{l}\text { Agenda } 21 \text { para la industria de } \\
\text { los viajes y el turismo }\end{array}$ & 1995 & OMT & \\
\hline $\begin{array}{l}\text { Conferencia Internacional sobre } \\
\text { Biodiversidad y Turismo }\end{array}$ & 1997 & $\begin{array}{l}\text { UE, ONU, } \\
\text { OMT, UICN }\end{array}$ & $\begin{array}{l}\text { Berlín } \\
\text { (Alemania) }\end{array}$ \\
\hline $\begin{array}{l}\text { La carta europea de turismo en } \\
\text { áreas protegidas }\end{array}$ & 1997 & Europarc & \\
\hline $\begin{array}{l}\text { Declaración sobre el turismo y el } \\
\text { desarrollo sostenible }\end{array}$ & 1997 & OMT & Bali \\
\hline $\begin{array}{l}\text { Conferencia sobre Empleo y } \\
\text { Turismo }\end{array}$ & 1997 & UE & Luxemburgo \\
\hline Código ético del turismo mundial & 1999 & OMT & $\begin{array}{l}\text { Santiago de Chile } \\
\text { (Chile) }\end{array}$ \\
\hline $\begin{array}{l}\text { Carta internacional } \\
\text { turismo cultural }\end{array}$ & 1999 & OMT & \\
\hline 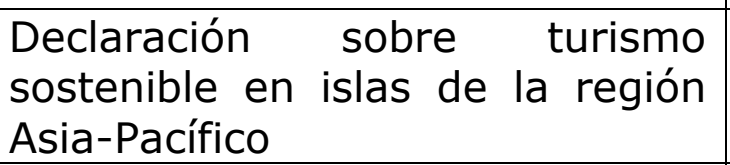 & 2000 & OMT & \\
\hline la Carta del Turismo Sostenible & 2001 & OMT & $\begin{array}{l}\text { Rímini } \\
\text { (Italia) }\end{array}$ \\
\hline
\end{tabular}




\begin{tabular}{|l|l|l|l|}
\hline $\begin{array}{l}\text { Red card for tourism? Ten } \\
\text { principles and challenges for a } \\
\text { sustainable tourism development } \\
\text { in the 21 st century }\end{array}$ & $\begin{array}{l}\text { Red para el } \\
\text { desarrollo } \\
\text { sostenible del } \\
\text { turismo } \\
\text { (DANTE) }\end{array}$ & $\begin{array}{l}\text { Johannesburgo } \\
\text { (Sudáfrica) }\end{array}$ \\
\hline $\begin{array}{l}\text { Declaración sobre ecoturismo } \\
\text { UNEP, OMT, } \\
\text { Comisión } \\
\text { canadiense de } \\
\text { turismo }\end{array}$ & $\begin{array}{l}\text { Quebec } \\
\text { (Canadá) }\end{array}$ \\
\hline $\begin{array}{l}\text { Declaración sobre Turismo y } \\
\text { Cambio Climático }\end{array}$ & 2003 & OMT & Djerba (Túnez) \\
\hline $\begin{array}{l}\text { Tourism and biodiversity: } \\
\text { mapping tourism's Global } \\
\text { Footprint turismo }\end{array}$ & 2003 & UNEP & OMT \\
\hline $\begin{array}{l}\text { Declaración sobre } \\
\text { cultural y alivio de la pobreza }\end{array}$ & & & Hue (Vietnam) \\
\hline
\end{tabular}

Tabla 2 - Listado no exhaustivo de eventos y documentos relacionados con la sostenibilidad en el ámbito del turismo

Fuente: elaboración propia

En los años ochenta, las resoluciones contenidas declaraciones de Manila y Acapulco o en la Carta del Turismo Sostenible y el Código del Turista, destacaban la preocupación por el contexto social, económico, cultural y natural de los destinos. Mientras que la Conferencia Interparlamentaria sobre Turismo manifiesta que "la integridad del medio natural, cultural y humano es una condición fundamental para el desarrollo del turismo" (Marín y Mendaro, 1996).

Por su parte, en el congreso denominado Turismo Cualitativo, la Asociación Internacional de Expertos en Científicos del Turismo definía el turismo sostenible como: "aquel que mantiene un equilibrio entre los intereses sociales, económicos y ecológicos" (Citado en Valdés, 2004, pp. 169). Llegando a la conclusión de que el desarrollo turístico sólo podrá ser positivo mientras las necesidades de la población local sean situadas antes que los objetivos del negocio turístico (Instituto de Estudios Turísticos, 1997). 
Además de esto, puede destacarse una multitud de recomendaciones y directrices encaminadas a facilitar la sostenibilidad en los ámbitos de planificación y gestión o códigos éticos dirigidos a turistas.

(c) Este paradigma se ha integrado en la vida cotidiana, la política, la planificación y los negocios con gran intensidad (Lanfant y Graburn, 1994; Miller, 2003; Santana, 2008); hasta tal punto que los discursos referidos a la sostenibilidad son tan abundantes y se refieren a cosas tan diferentes que el término ha perdido su significado (Butler, 1994; De Kadt, 1994).

Así pues, cuestiones como sostenibilidad, ecología, importancia de la conservación de la naturaleza o la diversidad cultural, cambio climático, etc. han sido incorporadas al imaginario de los ciudadanos (especialmente en los países industrializados) con implicaciones importantes en el ámbito del turismo. Tanto las motivaciones para el viaje como los comportamientos del turista han cambiado ante el flujo de información referente a los efectos negativos generados por el desarrollo del turismo en su forma tradicional.

De acuerdo con esto, en ciertos ámbitos, la forma de turismo convencional que surge masivamente tras la Segunda Guerra Mundial pierde prestigio social, siendo denostado por muchos. Estableciéndose incluso una distinción entre turistas y viajeros (estando los primeros ligados al turismo masivo, normalmente con motivaciones relacionadas con el descanso y la recreación en playas soleadas y adaptadas para su uso turístico masivo y los segundos con otras formas de turismo, normalmente de pequeños grupos, en espacios aparentemente menos adaptados para la actividad turística, interesados en el contacto cultural y la observación de lo natural) que aumenta las diferencias de clase entre los usuarios del sistema turístico (Urry, 1996; Rodríguez Regueira, 2001).

Si bien, hay que matizar que el turismo siempre ha estado determinado por la estructura social de clases; ya que (a) en la prehistoria del turismo de masas éste era realizado únicamente por determinadas élites, (b) en los años de desarrollo de lo que podemos denominar turismo convencional la distinción social, entre aquella minoría de la población mundial con acceso al turismo, se 
materializaba en la elección del destino (cuanto más lejano mayor nivel social), en el transporte (tren, automóvil, avión, etc. Con diferentes categorías interiores en el caso del tren o el avión), en el alojamiento (por ejemplo a través del sistema de estrellas en los hoteles), en las actividades realizadas en el destino (que cubren un amplio espectro desde beber cerveza en un chiringuito de playa hasta jugar a golf en un hotel de lujo), etc.

Parece que es a partir de los años noventa cuando aumentan las diferencias entre turistas. Siguiendo a MacCannell (2003), mofarse de los turistas convencionales confiere cierta elegancia intelectual; "la palabra turista se utiliza cada vez más como una etiqueta despectiva para alguien que parece satisfecho con sus experiencias que, evidentemente, no son auténticas" (MacCannell, 2003: 125).

En oposición a los denostados turistas convencionales aparecen los nuevos turistas o post-turistas (Harkin, 1995; Cohen, 1996b, 2005; Wang, 1999; Galani-Moutafi, 2000; Tucker, 2001; Santana, 2003, 2008; Hampton, 2005). Que ya no se conforman con la contratación de paquetes estandarizados (García Henche, 2005) en entornos evidentemente adaptados para la actividad turística $y$, por lo tanto, degradados; demandan ofertas individualizadas en entornos aparentemente poco adaptados (si bien con las mismas comodidades que en el entorno de origen), desean sentirse diferentes y superiores a los turistas convencionales, alcanzar una apreciación más profunda del mundo (Harkin, 1995; Cohen, 1996b, 2005; Wang, 1999; GalaniMoutafi, 2000; MacCannell, 2003; Barretto, 2007; Duterme, 2007). Utilizando el turismo como una forma de identificarse y distinguirse de otros de igual forma que los complementos de moda (Frangialli, 1998).

Siguiendo lo planteado por Cohen (2005), estos nuevos turistas están determinados por la búsqueda de tres experiencias principales:

1. La distinción; buscada por individuos que poseen un grado relativamente alto de capital cultural, sofisticación y reflexividad. Que han descubierto que las motivaciones de los nuevos turistas son, nuevamente, una ilusión. Conscientes de que lo exótico es siempre simulación, prefieren practicar 
turismo en espacios lujosos y cercanos antes que buscar lo extraordinario en entornos evidentemente no auténticos.

2. La fantasía; ante la experiencia de homogeneización del mundo, prefieren visitar espacios de fantasía (principalmente parques temáticos) y formar parte de la ficción.

3. Lo extremo; es practicado por individuos que buscan la excitación, la emoción y el riesgo del viaje en aquellos lugares de la Tierra que aún permanecen relativamente "salvajes".

De acuerdo con lo planteado por Cohen, en el mundo postmoderno pueden diferenciarse aquellos turistas que aparecen por oposición a los turistas de convencionales y otros que surgen por oposición a los anteriores. Es decir, ante la difusión de los discursos que llaman la atención sobre los aspectos negativos de las formas de turismo que surgen tras la Segunda Guerra Mundial, aparecen turistas interesados en el contacto auténtico con otras culturas y espacios naturales relativamente salvajes; pero es que, a partir de la constatación de la homogeneización del mundo y la percepción de la búsqueda de la autenticidad como ideal de la modernidad, surge un nuevo grupo de turistas que, en oposición con los anteriores, prefieren visitar entornos cercanos (con prestaciones de alta calidad) o inmiscuirse en fantasías que se muestran abiertamente como ficción.

Sin embargo, no puede perderse de vista el hecho de que, si bien estas tendencias pueden tener importancia, los turistas siguen siendo mayoritariamente convencionales y la demanda de paquetes turísticos estandarizados y destinos evidentemente modificados para su explotación turística masiva continúa siendo relevante (Lickorish y Jenkins, 2000). De tal forma que turistas y post-turistas conviven en la actualidad, si bien podrían plantearse diferencias en relación con el entorno socioeconómico y cultural de unos y otros.

La diversificación de motivaciones e intereses de los turistas unida a la difusión entre empresarios, planificadores y gestores de los efectos negativos atribuibles a las formas convencionales de turismo, ha conllevado 
modificaciones en la oferta, la segmentación del mercado y la aparición de una multitud de nuevas formas de turismo (Eadington y Smith, 1994; Smith, 2001a; García Sánchez y Albuquerque García, 2003; Santana, 2003, 2008; Valdés, 2004; Avilá Bercial y Barrado Timón, 2005; Bushell, y Eagles, 2007; Cruz Vareiro y Cadima Ribeiro, 2007). Apareciendo nuevos productos (presentados como sostenibles, responsables, ecológicos, solidarios, naturales, etc.), destinos (cuyas características no atraían al turismo convencional) y un elenco cada vez más influyente de estándares de calidad ambiental y sostenibilidad (Buckley, 2002; Miller, 2003).

La feroz competencia entre destinos y empresas por atraer turistas ha jugado un papel importante en la segmentación del mercado y la aparición de las "nuevas formas de turismo" (Eadington y Smith, 1994). El interés por obtener un mejor posicionamiento les ha llevado, a partir de la década de los setenta, a la aplicación de estrategias de marketing basadas en la sostenibilidad (Mastny, 2003; Miller, 2003). Siendo defendible la idea de que los agentes encargados de generar imagen para su venta turística han reforzado las nuevas tendencias dando solidez, cuando no generando, las necesidades emergentes en relación con la sostenibilidad.

\section{Conclusiones}

El turismo ha pasado por múltiples modificaciones desde su aparición, pero las surgidas en la década de los 90 fueron de especial interés, en tanto que se presentaron como productos específicos y se introdujeron en las mentalidades de sus potenciales clientes a través de criterios más de carácter moral que económico. La salvaguarda futura del entorno y sus culturas, la manifestación de las conciencias individuales, la liberación y realización personal a través de choques de adrenalina y experiencias, entre otras, se incorporaron a la cotidianeidad, cuando antes eran un extraordinario. Es precisamente ésta la gran modificación de la actividad turística y del propio sistema iniciado en los noventa del pasado siglo y consolidado en la actualidad. 
No es cuestión de plantear el dilema del "huevo y la gallina", "la oferta y la demanda". Procesos que derivaron del cambio de paradigma de la sociedad occidentalizada, abrumada por los cambios del economicismo y que, prácticamente, abría los ojos al cambio climático, la destrucción de la naturaleza, la homogeneización supuesta de las grandes urbes, etc. Se imbricaron con los intereses de brokers culturales, innovadores empresariales, líderes de opinión y algunos medios de comunicación que contribuyeron en gran medida a generalizar los cambios mientras se construían, casi por ensayo y error, aquellos nuevos productos y destinos. El modelo, sin abandonar los planteamientos hedonistas anteriores en torno a las vacaciones, llegó para quedarse.

Dando continuidad, el mercado está abierto a productos especiales, vendidos como únicos, en los que la vivencia de experiencias al límite constituye su centro. Convertirse, por un periodo vacacional y bajo condiciones de seguridad, en emigrante ilegal (la simulación de cruzar la frontera entre Méjico y EEUU, incluyendo una ficción de persecución y secuestro policial), director de cine pornográfico (la observación, desde la silla anexa a la del director, del rodaje de una película de este género) o superviviente de una catástrofe (la visita a lugares devastados por la guerra o fenómenos naturales), son una pequeña muestra de la adaptabilidad y dinamismo del sistema. Así, concluyendo, la cuestión se desplaza de qué puedo ofertar a qué desea el cliente potencial.

\section{Bibliografía}

Avilá Bercial, R., \& Barrado Timón, D. A. (2005). Nuevas tendencias en el desarrollo de destinos turísticos: Marcos conceptuales y operativos para su planificación y gestión. Cuadernos de Turismo, n.15, pp.27-43.

Barretto, M. (2007). Turismo y cultura; relaciones, contradicciones $y$ expectativas. Online: Pasos Edita.

Butler, R. (1994). Alternative tourist; the thin edge of the wedge. In V. Smith, \& W. Eadington (Eds.), Alternatives tourism (pp.31-46). Chichester: John Wiley \& sons. 
Ceballos-Lascuráin, H. (1996). Tourism, ecotourism and protected areas. Switzerland and Cambridge: IUCN.

Cohen, E. (1996(a)). The sociology of tourism; approaches, issues, and findings. In Y. Apostolopoulos, S. Leivadi \& A. Yiannakis (Eds.). The sociology of tourism; theoretical and empirical investigations. (pp.51-71). London: Routledge.

Cohen, E. (1996(b)). A phenomenology of tourist experiences. In $Y$. Apostolopoulos, S. Leivadi \& A. Yiannakis (Eds.). The sociology of tourism; theoretical and empirical investigations. (pp.90-111). London: Routledge.

Comisión Mundial sobre el Medio Ambiente y el Desarrollo. (1989). Nuestro futuro común. Madrid: Alianza.

Cruz Vareiro, L., \& Cadima Ribeiro, J. (2007). Sustainable use of endogenous touristic resources of rural areas: Two portuguese case studies. Pasos Revista de Turismo y Patrimonio Cultural. Vol. 5, N.2, 193-207.

De Kadt, E. (1994). Making the alternative sustainable: Lessons from development for tourism. In V. Smith, \& W. Eadington (Eds.). Alternatives tourism (pp.47-75). Chichester: John Wiley \& sons.

Drumm, A., \& Moore, A. (2002). Desarrollo del ecoturismo; un manual para los profesionales de la conservación, vol.1. Virginia, EEUU.: The nature Conservancy.

Duterme, B. (2007). Turismo hoy: Ganadores y perdedores. In VVAA (Ed.), Turismo hoy: Ganadores y perdedores (pp.17-26). Madrid: Popular.

Eadington, W., \& Smith, V. (1994). Introduction: The emergence of alternative forms of tourism. In V. Smith, \& W. Eadington (Eds.). Tourism alternatives. Chichester: John Wiley \& sons.

Epler Wood, M. (2002). Ecotourism: Principles, practices y policies for sustainability. www.ecotourism.org: UNEP.

Frangialli, F. (1998). Mirando la bola de cristal. Revista Valenciana d'Estudis Autonomics.

Galani-Moutafi, V. (2000). The self and the other. Traveler, ethnographer, tourist. Annals of Tourism Research, 27 (1), pp.203-224.

García Henche, B. (2005). Características diferenciales del producto turismo rural. Cuadernos de Turismo, n.15, pp.113-133.

García Sánchez, A., \& Albuquerque García, F. J. (2003). El turismo cultural y el de sol y playa ¿sustitutivos o complementarios? Cuadernos de Turismo, n.11.

Gimeno, J. C., \& Monreal, P. (1999). La controversia del desarrollo Catarata. Hampton, M. (2005). Heritage, local communities and economic development. Annals of Tourism Research, Vol 32, n.3, pp.735-759.

Harkin, M. (1995). Modernist antrophology and tourism of the authentic. Annals of Tourism Research, 22 (3), pp.650-670.

Hernandez Cruz, R., Bello Baltazar, E., Montoya Gómez, G., \& Estrada Lugo, E. (2005). Social adaptation; cotourism in the lancandon forest. Annals of Tourism Research, Vol 32, n.3, pp.610-627.

Jafari, J. (1994). La cientifización del turismo. Estudios y Perspectivas en Turismo, 3(1): pp.7-36. 
Lanfant, M., \& Graburn, N. (1994). International tourism reconsidered: The principle of the alternative. In V. Smith, \& W. Eadington (Eds.). Alternatives tourism (pp. 88-112). Chichester: John Wiley \& sons.

MacCannell, D. (2003). El turista, una nueva clase ociosa. Barcelona: melusina.

Manwa, H. (2003). Widlife-based tourism, ecology and sustainability: a tug-ofwar among competing interests in Zimbabwe. The Journal of Tourism Studies, Vol 14, n.2, pp.45-54.

Marín, C., \& Mendaro, C. (1996). Turismo y desarrollo sostenible: El desafío ante el siglo XXI. Los casos de Lanzarote, Menorca y Sierra de las Nieves. Madrid: Comité Español del Programa MaB.

Mastny, L. (2003). Ecoturismo: Nuevos caminos para el turismo internacional. Bilbao: Bakeaz.

Miller, G. (2003). Consumerism in sostainable tourism: a survey of UK consumers. Journal of Sustainable Tourism, Vol. 11, N.1, pp.17-39.

Mora, A., Duch Devesa, M., \& Córdova Leiva, J. (2001). El desarrollo del turismo en el parque natural del alto tajo. Cuadernos De Turismo, n.7, pp.111130.

Puertas Cañaveral, I., \& Paniza Prados, J. L. (2007). Ecoturismo, sostenibilidad y comunidad local. Turismo y sociedad en Andalucía (pp. 307-332). Andalucía: Junta de Andalucía, Consejería de Turismo, Comercio y Deporte.

Rodríguez Regueira, J. L. (2001). La reinvención cosmopolita de la autenticidad. www. Naya.Org.ar/turismo,

Salinas Chávez, E. \& La O Osorio, J. A. (2006). Turismo y sustentabilidad: de la teoría a la práctica en Cuba. Cuadernos de Turismo, n.17, pp.201-221.

Santana Talavera, A. (2003). Patrimonios culturales y turistas: unos leen lo que otros miran. Pasos Revista de Turismo y Patrimonio Cultural, 1(1), 1-12.

Santana Talavera, A. (2008). El turismo cultural: ¿un negocio responsable? Paper presented at the 272-294.

Santana Talavera, A. (2009). Antropología del turismo; analogías, encuentros y relaciones.

Santana Turégano, M. (2007). Turismo, economía y planficación urbana: Una relación compleja. Pasos Revista de Turismo y Patrimonio Cultural. Vol.5, N.1, 53-67.

Smith, V. (2001(a)). The nature of tourism. In V. Smith, \& M. Brent (Eds.), Host and guest revisited: Tourism issues of the 21st century (pp. 53-68). Nueva York: Cognizant Communication Corporation.

Smith, V. (2001(b)). Tourism change and impacts. In V. Smith, \& M. Brent (Eds.), Host and guest revisited: Tourism issues of the 21st century (pp. 107121). Nueva York: Cognizant Communication Corporation.

Smith, V. (2001(c)). Sustainability. In V. Smith, \& M. Brent (Eds.), Host and guest revisited: Tourism issues of the 21st century. Nueva York: Cognizant Communication Corporation.

Stronza, A., \& Gordillo, J. (2008). Community views of ecotourism. Annals of Tourism Research, 35 (2), pp.448-68. 
Tucker, H. (2001). Tourists and troglodytes. Annals of Tourism Research, 28 (4), 868-891.

Turismo y desarrollo sostenible: Aproximación conceptual y documental(1997). Madrid: Instituto de Estudios Turísticos.

Urry, J. (1996). Tourism, culture and social inequality. In Y. Apostolopoulos, S. Leivadi \& A. Yiannakis (Eds.). The sociology of tourism; theoretical and empirical investigations (pp.115-133). London: Routledge.

Valdés, L. (2004). Turismo sostenible y turismo rural. In E. Uriel, \& R. Hernández (Eds.), Análisis y tendencias del turismo (pp. 165-168) Pirámide.

Vidaurreta Campillo. (2003). Conservación. Ecosistemas, áreas protegidas y factores socioculturales. Madrid: UNED.

Viola Recasens, A. (. ). (2004). Antropología del desarrollo. Barcelona: Paidós. Wang, N. (1999). Rethinking authenticity in tourism experience. Annals of Tourism Research, 26 (2), 349-370.

Artigo recebido em novembro de 2010.

Aprovado para publicação em novembro de 2010. 